\title{
A novel Multi-Phase Flash Sintering (MPFS) technique for 3D complex-shaped ceramics
}

\author{
Sandra Molina-Molina a,1, Eva Gil-González ${ }^{a, b, 1, *}$, Francisco José Durán-Olivencia ${ }^{\mathrm{c}}$, \\ José Manuel Valverde ${ }^{\mathrm{d}}$, Antonio Perejón ${ }^{\mathrm{a}, \mathrm{e}}$, Pedro E. Sánchez-Jiménez ${ }^{\mathrm{a}, \mathrm{e}, *}$, \\ Luis A. Pérez-Maqueda ${ }^{\mathrm{a}, *}$ \\ a Instituto de Ciencia de Materiales de Sevilla, Consejo Superior de Investigaciones Científicas-Universidad de Sevilla, Calle Américo Vespucio 49, Sevilla \\ 41092, Spain \\ b Departamento de Ingeniería Química, Universidad de Sevilla, Escuela Politécnica Superior, Calle Virgen de África, 7, Sevilla 41011, Spain \\ ' Departamento de Ingeniería, Universidad Loyola Andalucía, Avda. de Las Universidades s/n, Sevilla 41704, Spain \\ d Departamento de Electrónica y Electromagnetismo, Facultad de Física, Universidad de Sevilla, Avda. Reina Mercedes s/n, Sevilla 41012, Spain \\ e Departamento de Química Inorgánica, Facultad de Química, Universidad de Sevilla, Avda. Reina Mercedes s/n, Sevilla 41012, Spain
}

\section{A R T I C L E I N F O}

\section{Article history:}

Received 13 September 2021

Revised 4 November 2021

Accepted 11 November 2021

\section{Keywords:}

Flash sintering

Alternating current

Field-assisted sintering techniques

Complex shape

Ceramic materials

Yttria-stabilized zirconia

\begin{abstract}
A B S T R A C T
This work demonstrates the first proof-of-concept of Multi-Phase Flash Sintering (MPFS). This novel technique essentially consists of applying a rotating electric field to the sample by means of a multi-phase voltage source as furnace temperature increases. Several ceramic materials with different types of electrical conductivities are sintered within seconds at furnace temperatures much lower than those used for traditional DC flash sintering due to the higher power densities administered by a multi-phase power supply. Thus, ceramic materials are flashed at relatively lower applied voltages which minimizes undesired phenomena such as localization and preferential current pathways. Furthermore, MPFS allows diverse electrode configurations to promote a more uniform electric field distribution, enhancing the sintering of 3D complex-shaped specimens. MPFS could be a true breakthrough in materials processing, as 3D complex-shaped specimens are homogeneously sintered at reduced temperatures, while keeping all the advantages of conventional flash sintering.
\end{abstract}

(c) 2021 The Author(s). Published by Elsevier Ltd. This is an open access article under the CC BY-NC-ND license (http://creativecommons.org/licenses/by-nc-nd/4.0/)

\section{Introduction}

Since its inception in 2010, Flash Sintering (FS) has become one of the most exciting of the Field-Assisted Sintering Techniques (FAST) [1]. Rishi Rajś group at Colorado University first observed that $\mathrm{ZrO}_{2}$ could be sintered in just a few seconds at greatly reduced temperatures by applying an external electrical field while the sample is being heated [2]. In a typical flash experiment, a set voltage is applied allowing the current to flow through the sample while the temperature is linearly increased. The electrical conductivity of the ceramic rises with temperature which leads to an amplification of the current intensity traversing the specimen. At a certain temperature, the sample enters the so-called "flash" stage, revealed by a sudden drop in the electrical resistivity and a

\footnotetext{
* Corresponding author.

E-mail addresses: eva.gil@icmse.csic.es (E. Gil-González), pedro.enrique@ icmse.csic.es (P.E. Sánchez-Jiménez), maqueda@cica.es (L.A. Pérez-Maqueda).

1 These authors contributed equally to this work as first authors.
}

surge in the power density dissipated by the sample. At this critical point, the current intensity must be quickly controlled to the preset value, which is maintained for a given period of time. The flash event is generally accompanied by intense photoluminescence as well as almost instantaneous densification [3]. It is now accepted that the flash onset temperature is inversely related to the amplitude of the applied electric field [4]. Probably, the most advantageous feature of FS is its environmental-friendly nature due to the energy and time saving in comparison to conventional procedures. FS has been demonstrated for many ceramic materials, exhibiting different electrical conductivities, and all of them characterized by a negative temperature coefficient of resistivity $[5,6]$.

Intensive research has been devoted not only to the application of FS to conventional and advanced ceramic materials but also to the understanding of the flash phenomena. However, the fundamental mechanisms that govern FS remain elusive. Several possibilities have been proposed, such as Joule heating [7], preferen- 
tial Joule heating at grain boundaries leading to local melting $[8,9]$ and the formation of defects and new species such as Frenkel pairs and electrochemically reduced species, respectively [2,10-12]. Abnormal lattice expansion has also been observed by in-situ EnergyDispersive X-Ray Diffraction [13,14]. Nevertheless, none of them can solely explain by itself the FS phenomena and it is quite likely the simultaneous implication of several of the proposed mechanisms during flash [7]. On the other hand, another important body of work has been committed to exploit the capabilities of the technique. FS promotes the sintering of thermally unstable or hard-tosinter materials, such as $\mathrm{BiFeO}_{3}$ and related materials [15-18] or garnet-type solid state electrolytes [19]. In some cases, the specimens are granted with unique properties, such as superplasticity in $\mathrm{TiO}_{2}$ [20] and enhanced catalytic properties in $\mathrm{SrTiO}_{3}$ [21]. Additional efforts have been devoted to exploit the eco-friendliest side of FS, such as further reducing the flash onset temperature by applying strong electric fields (higher than $1000 \mathrm{~V} \mathrm{~cm}^{-1}$ [22]) or using water or reductive atmospheres [23,24]. Moreover, it has been recently developed the so-called "Reactive-Flash Sintering", which merges sintering and chemical reaction in a single step [25]. This technique has been successfully applied to the preparation and processing of advanced ceramics with complex stoichiometry [2629].

Despite the new opportunities brought about by FS, a few issues must be overcome for practical implementation. Some of them are related to the thermal management during Flash that leads to heterogeneities of different natures (such as abnormal grain growth, redox reaction at the electrodes or local melting [3032]) due to the formation of preferential current paths promoting thermal gradients that are amplified by cooling fluxes at the surface of the samples, leading to the formation of hot spots [33]. The formation of those hot spots depends on several factors such as the sample geometry, properties of the materials, electrodes composition and configuration as well as the employed experimental conditions. Note that this is precisely the reason of the small cross section of the dog bone samples typically used in FS, as this shape minimizes the gradients between the core and the surface of the samples. Nevertheless, several strategies have been developed to alleviate the effects of these thermal management issues in FS. For instance, the operational conditions can be carefully controlled in order to avoid the common power spike in conventional FS by running isothermal experiments with different controlled current ramp profiles [34-36]. Ultrahigh heating rates and the use of thermal insulators to minimize the heating cavity air volume in FS setups, have been reported to promote homogeneous heating by minimizing cooling fluxes and heat loses at the surface of the specimen [37]. The redox reactions at the electrodes, amply reported in ionic conductors such as zirconia, can be partially mitigated by a suitable electrode composition and configuration [38-40]. The addition of electrical conductive additives to ceramic matrix also decreases the probability of hot spots formation but, on the other hand, can detrimentally modify the final properties of the materials [41,42]. Moreover, sintering homogeneity can be generally enhanced by applying AC fields, although the effect of frequency remains controversial. In spite of these efforts, none of these strategies or the combination of several of them seem to be a universal approach to overcome the thermal management issues and the practical implementation of FS. Additionally, another burden for practical applications is that finding the optimum combination of electrical field amplitude and maximum current density leading to a dense and homogeneous sample is currently more art than science, as this pair normally lies within a very limited range of applied power $[4,26]$. Yet, probably the most important obstacle for the practical implementation of FS is that the experimental setup is limited to two electrodes. This restricts the specimen shapes to dog-bones, rods, cylinders, plates [12] (and very recently thick and thin films $[43,44]$ are also under investigation), which unavoidably constrains the technological applications. Furthermore, localization and preferential current path issues are exacerbated in these limited configurations, as the current flow is necessarily either uni- or bi-directional. Therefore, a true breakthrough in materials processing would be the development of a novel FS technique for sintering three dimensional (3D) samples with complex geometry, leading to substantial energy and time saving among the many other benefits of FS. Moreover, the rapid growth of 3D-printing in the last few years would ease the possibility of manufacturing complexshaped samples which would expand the domain of applications of ceramic materials [45].

Here, we propose the application of a rotating electric field to enhance FS in ceramic materials. Under this configuration, three electrodes (or more) are equidistantly placed over the edges of the sample and connected to a multi-phase power supply, creating a rotating electric field across the entire sample. It is shown that 3D complex-shaped specimens are homogeneously sintered within seconds at furnace temperatures much lower than those used in conventional DC-FS. This novel Multi-Phase Flash Sintering (MPFS) technique has been successfully applied to several materials with different electrical conductivities such as $8 \mathrm{~mol} \%$ yttriastabilized zirconia (8YSZ), $\mathrm{BiFeO}_{3}$ (BFO) and $\mathrm{ZnO}$, proving the wide applicability of the technique. It is noteworthy that quite recently an attempt to sinter a complex-shaped gear by traditional DC-FS has been made by applying a DC voltage between the two faces of the green body [46]. Nevertheless, this approach requires of a specimen with two flat parallel surfaces and it has been observed grain size and hardness variations along its geometry. As shown in the present work, this serious issue is overcome with the proposed MPFS technique, which leads to a uniform distribution of the electric field across the material. Different types of multi-phase electrodes configurations can be disposed in convoluted arrangements for complex geometries while all the advantages of traditional FS are preserved. All in all, the proposed MPFS methodology can constitute a true breakthrough in ceramics processing, opening the venue to innovate multi-phase electrodes configurations and fostering the technological applications of Flash-Sintered materials with 3D-structures.

\section{Materials and methods}

\subsection{Materials and characterization}

Commercial powders of $8 \mathrm{~mol} \%$ yttria-stabilized zirconia (8YSZ) (Tosoh Corporation, TZ-8Y) and ZnO (Sigma-Aldrich, 544,906, nanopowder, $<100 \mathrm{~nm}$ ) were directly used, whereas BFO powders were prepared by mechanosynthesis. To this end, stoichiometric amounts of $\mathrm{Fe}_{2} \mathrm{O}_{3}$ (Sigma-Aldrich, 310,050, < $5 \mu \mathrm{m}$, $\geq 99 \%$ ) and $\mathrm{Bi}_{2} \mathrm{O}_{3}$ (Sigma-Aldrich, 223,891, $10 \mu \mathrm{m}, 99.9 \%$ ) were milled according to the procedure described by the reference herein [47]. To make the pellets, the powders were mixed by a $5 \% \mathrm{w} / \mathrm{w}$ with a 5\% PVA solution in distilled water (Sigma-Aldrich, 363,138, 98.0$98.8 \%$ ). The mixtures were dried overnight at $80{ }^{\circ} \mathrm{C}$ and uniaxially pressed at $250 \mathrm{MPa}$ into $10 \mathrm{~mm}$ diameter and $1.5 \mathrm{~mm}$ thickness disk-shaped pellets. 3D complex-shaped samples (triangle and clover) were obtained from $20 \mathrm{~mm}$ diameter cylindrical pellets of $8 Y S Z$, which were cut into the desired shape. Details about the dimensions of these green bodies are shown in Fig. S1. The binder was removed by heating the green compacts at $500{ }^{\circ} \mathrm{C}$ for $30 \mathrm{~min}$.

The density of the samples was measured by Archimedes' method using distilled water at room temperature as the immersion liquid. Microstructural characterization was carried out using a Scanning Electron Microscopy (SEM) Hitachi S-4800 SEM-FEG operated at $2 \mathrm{kV}$. 


\subsection{Experimental setup}

The MPFS setup is similar to those used in conventional DC-FS and basically consists of a furnace, camera, computer, power supply and power analyzer. A schematic representation is depicted in Scheme 1(a). The main differences with respect to a conventional FS setup lie on the employed power supply. MPFS tests use a 3phase AC voltage source. The samples are connected to the power supply using two basic configurations: Delta and Star (also known as $\mathrm{Y} / \mathrm{Wye}$ ), which are schematically represented in Scheme 1(b) and (c), respectively. Delta configuration wiring consists of three wires or phases equidistantly placed (at $120^{\circ}$ for cylindrical samples) at the edges of the samples. Each wire is connected to the adjacent ones through their endpoints forming a triangle, as shown in Scheme 1(b). This configuration is a 3-phase 3-wire system and a particular phase by itself serves as a return line for the others. Alternatively, the Star configuration is a 3-phase 4-wire system. The 3 phases are connected to a common point where the neutral wire is attached, similarly to a wye. To this end, a hole is drilled in the geometric center of the sample as shown in the photograph of Scheme 1(c).

In order to perform the MPFS experiments, the samples were connected to a 3-phase AC power supply (AST1503A1 AMETEK Asterion, USA) in either Delta or Star configuration using platinum paste as electrodes and $\mathrm{NiCr}$ wires. A tubular furnace was employed for heating up the samples at a constant rate of $5{ }^{\circ} \mathrm{C} \mathrm{min}-1$ by means of a Eurotherm 3216 PID controller. A k-type thermocouple in the vicinity of the sample monitored the local temperature. Simultaneously, a rotating electric field was applied using three AC voltage signals of same amplitude and frequency $(50 \mathrm{~Hz})$ and phase shifted by $120^{\circ}$. The power supply was operated in a voltage-controlled mode until the flash event took place. At this critical point, the power supply automatically switched to currentcontrolled mode to counteract the abrupt non-linear rise of the conductivity. The flash event was maintained for a certain time lag before the power supply was turned off. The applied electric field and current density were recorded with a 3-phase power analyzer (PPA1500 Newtons4th Ltd, UK). Material densification and temperature were monitored using an infrared camera (PI 1M, Optris $\mathrm{GmbH}$, Germany). Additionally, optical images were recorded by means of a high-definition digital camera (ELP-USBFHD08S-MFV (5-50), ELP).

\section{Results and discussion}

Firstly, the two conventional connections of 3-phase systems, Delta and Star, were assessed. FS experiments were performed in cylindrical pellets of 8YSZ using both Delta and Star configurations. Fig. 1 shows an example of the total power density profile generated in Delta configuration. Similar trends and stages were obtained with Star-connected samples. This profile is the sum of the power generated by each one of the three phases, which can be seen in Fig. S2 (along with the total applied voltage and electrical current curves), divided by the total volume of the sample. As for single-phase FS, the three stages commonly categorized are also observed in MPFS (Fig. 1): incubation, transient and steady state (a.k.a. Stage I, II and III, respectively) [48]. During the incubation period there is a linear increase of electrical conductivity with temperature, which leads to a rise of the dissipated power. This stage culminates in the flash event, clearly identified by a non-linear increase of conductivity and a spike in the dissipated power. It is currently accepted that the flash is triggered by a thermal runaway [49]. The amount of generated heat overcomes the heat that can be dissipated by the specimen and that considerable increase of temperature leads to a great rise of the conductivity in a self-feed process [50]. At this point, the power supply is automat-

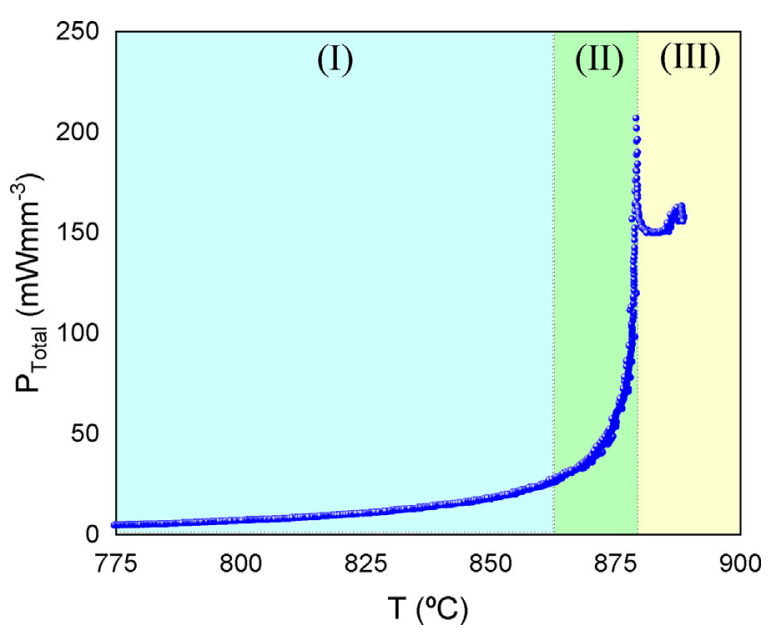

Fig. 1. Total power density profile as a function of furnace temperature generated during MPFS of a cylindrical 8YSZ pellet connected to the power supply in Delta configuration.

ically switched from voltage to current controlled mode to regulate the electrical runaway to the preset current limit (see also Fig. S2). Finally, the flash is maintained for $60 \mathrm{~s}$ under current-controlled mode, delivering constant power.

As mentioned above, both connections (Delta and Star) exhibit similar total dissipated power density trends. Simulations revealed almost the same electric field distribution in these two settings (Fig. S3). Detailed information about the simulations can be found in SI (1. Computational Simulations). The created rotating electric field for both configurations can be seen in Videos S1 and S2, where simulations over time are shown. For a given electric field distribution and temperature, it should not be expected too much difference between the two configurations in terms of conductivity, and, therefore, in the corresponding current densities. However, in Star-connected samples, current intensity spreads from phases to neutral while passing through smaller areas compared to Delta loads, where no neutral is required, and the current is driven from terminal to terminal. As a result, in Delta setups the current density goes through a larger effective area compared to Star connections. Therefore, as the current density passes through smaller effective areas in Star loads, it concentrates the local Joule heating in smaller regions, which eventually can trigger the flash selectively as higher conductivities are reached in those areas. Experimentally, a close examination of the intensity profiles in MPFS setups included in Fig. 2(a) and (b) shows that the Star connection brought forward the onset compared to the values registered in Delta configuration. This evidence is in good agreement with the mainstream about the thermal activation of the flash [49]. Additionally, Fig. 2(a) shows that the flash event is initiated simultaneously in the three phases under the Delta configuration. As confirmed by optical and IR thermographic images of the specimen during flash (Fig. 2(a) inset and 2(c), respectively), the flash quickly spreads throughout the whole geometry of the pellet, favoring a more homogeneous surface temperature distribution across the sample. This leads to more uniformly densified pellets, even for those with complex shape as will be shown ahead. Conversely, a time lag between the different phases is observed for the Star configuration (Fig. 2(b)). Phases 1 and 3 firstly flash, while flash of phase 2 is lagged. Flash phase delay is detrimental to achieve homogeneity and densification of the material (Fig. 2(b) inset). IR thermographic images (Fig. 2(d)) reveal significant temperature gradients across the surface of the sample, with differences of up to $200{ }^{\circ} \mathrm{C}$ between the areas where the flash has been initiated and those in which has not. Thus, the specimens cannot 

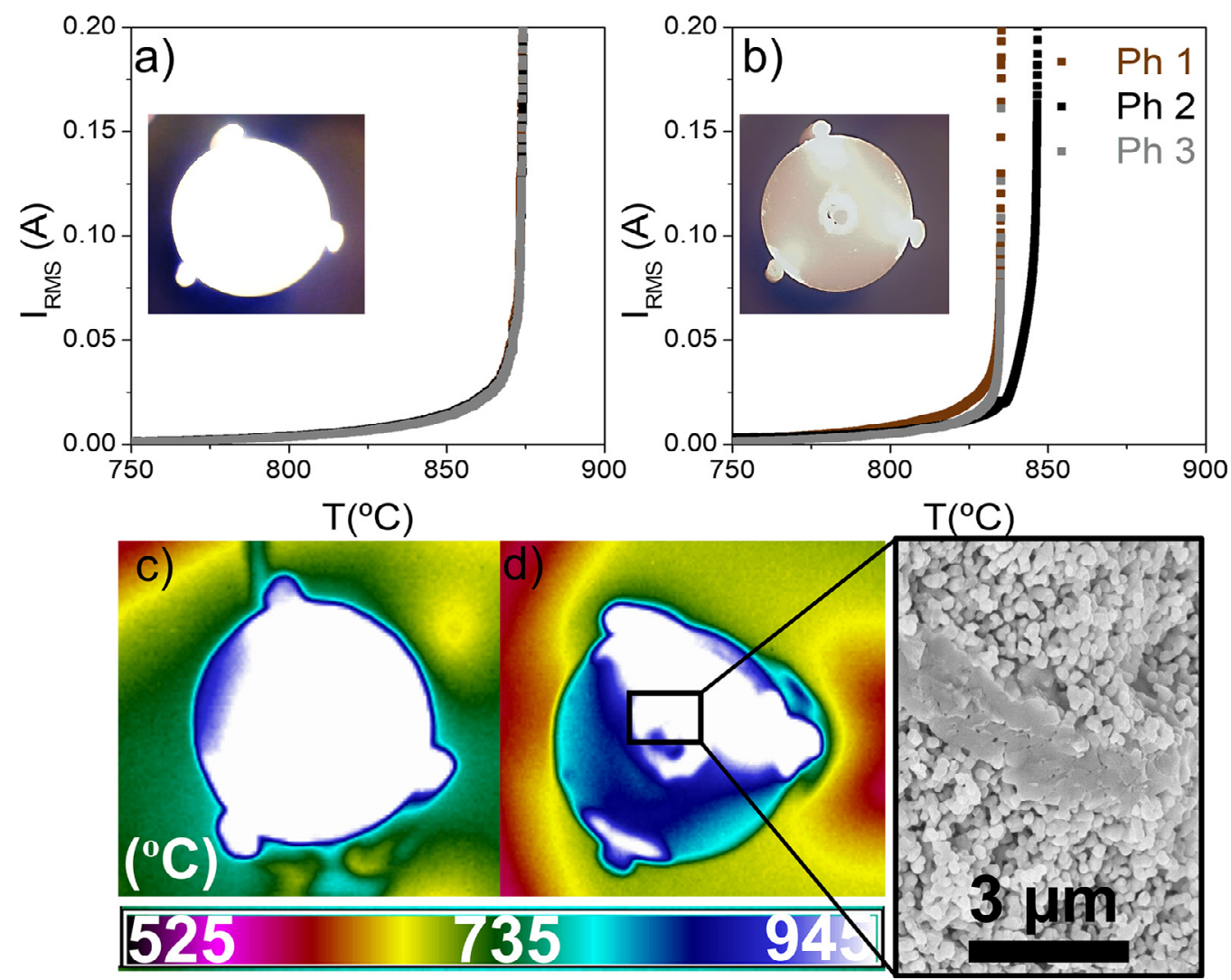

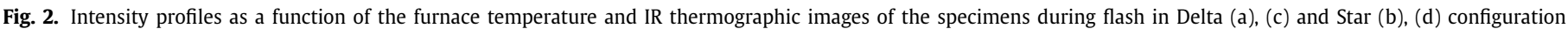

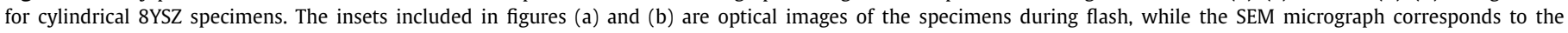
microstructure close to the neutral of a 8YSZ sample connected to the power supply in Star configuration.

be homogeneously densified. Indeed, this issue can be clearly observed by a simple visual inspection of the resulting specimens. As shown in Fig. S4, the obtained sintered samples by the Star configuration are significantly deformed. Additionally, heterogeneities in their microstructure are clearly seen in SEM images (Fig. 2). The SEM micrograph depicted in Fig. 2 was collected at low magnification in an area close to the neutral, where a melted region, probably caused by a preferential current path, along with poorly densified areas can be discerned. As inferred from computer simulations, Star-connected samples can experience a more significant local Joule heating, which may result in a localized non-uniform flash. These local effects can lead to the patterns reported in samples exposed to significant thermal gradients (i.e. blackening effect, porosity and inhomogeneous grain distribution, as well as abnormal grain growth $[33,38,51])$. A further disadvantage of the Star configuration is that it requires an extra wire to connect the neutral, which adds complexity and cost to scale-up the MPFS technology. Beside, an additional step in the sample preparation is needed, as a hole must be drilled in the center of the sample for the neutral connection. Therefore, Delta over Star configuration is preferred for MPFS systems. Experimental results hereafter reported correspond to Delta configuration tests.

Several materials with diverse types of conductivities (8YSZ, $\mathrm{ZnO}$ and BFO) were tested using the Delta configuration to assess the versatility and applicability of the MPFS technique (see also Fig. S5 that includes photographs of specimens before and after the MPFS as a way of examples). 8YSZ is an ionic conductor with almost negligible electronic conductivity, whereas $\mathrm{ZnO}$ and BFO are semiconductors presenting $n$-type and $p$-type conductivity, respectively. Fig. 3(a) shows the Arrhenius power density profile plots for $\mathrm{ZnO}$ and $\mathrm{BFO}$ at $20 \mathrm{~V}$ and for $8 \mathrm{YSZ}$ at $40 \mathrm{~V}$ using
Table 1

Activation Energies, $E_{a}$, obtained from the fit of Arrhenius curves to the data plotted in Fig. 3(b) for ZnO, 8YSZ and BFO under DC-FS and MPFS.

\begin{tabular}{lll}
\hline Material & System & $\left(\mathrm{E}_{\mathrm{a}} \pm \Delta \mathrm{E}_{\mathrm{a}}\right) \mathrm{eV}$ \\
\hline $\mathrm{ZnO}$ & DC-FS & $0.9094 \pm 0.0454$ \\
& MPFS & $1.0447 \pm 0.0519$ \\
$8 \mathrm{YSZ}$ & DC-FS & $0.3438 \pm 0.0123$ \\
& MPFS & $0.3671 \pm 0.0139$ \\
$\mathrm{BFO}$ & DC-FS & $0.4730 \pm 0.0543$ \\
& MPFS & $0.3833 \pm 0.0141$ \\
\hline
\end{tabular}

DC-FS and MPFS. Power profiles follow the typical expected behavior independently of the applied field and electrical properties of the materials. Thus, a linear increase of the power density is observed up to the flash onset, which is followed by an abrupt power spike. The flash event is triggered in a very narrow range of dissipated power density from about 6 to $30 \mathrm{~mW} / \mathrm{mm}^{3}$ as reported in previous works [4]. A remarkable feature of MPFS as compared to conventional DC-FS is that for the same applied voltage, at a given temperature the power delivered to the material is much higher. Consequently, the flash event is displaced to significantly lower temperatures, e.g. up to $80{ }^{\circ} \mathrm{C}$ in the case of BFO for an applied voltage of $20 \mathrm{~V}$. Fig. 3(b) shows the Arrhenius-type dependence of the applied voltage versus the flash onset temperature for ZnO, 8YSZ and BFO flashed under DC and 3-phase AC fields. The higher the applied voltage, the lower the flash onset temperature as typically observed in other works [52]. Interestingly, the Arrhenius-type dependence is almost parallel for the diverse materials. Thus, the activation energies (Table 1) are very similar. It is noteworthy that $8 Y S Z$ cannot be flashed under the conventional DC configuration below an applied voltage of $30 \mathrm{~V}$ (represented in 

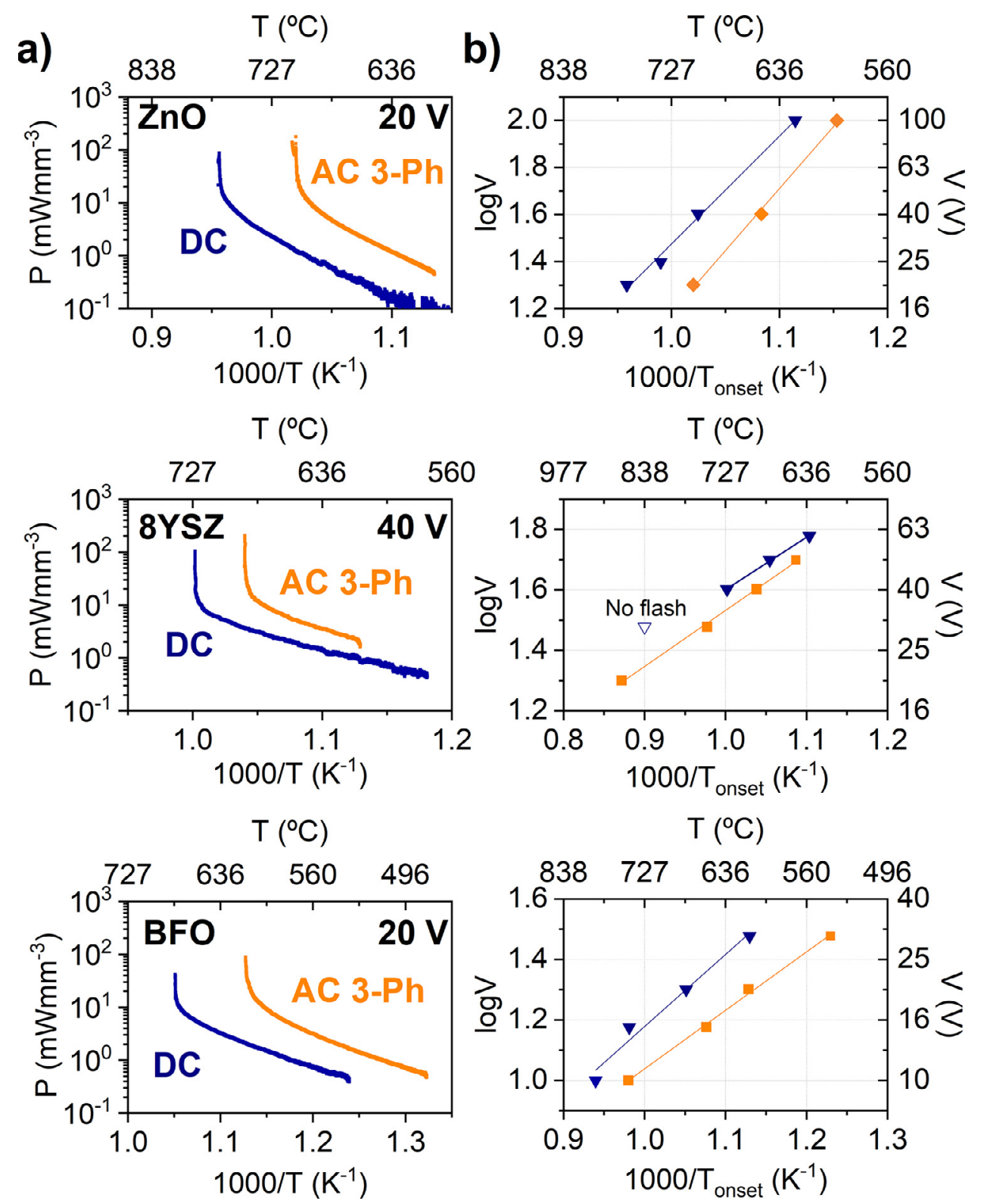

Fig. 3. (a) Power density and (b) applied voltage versus onset temperature (Arrhenius plots) for ZnO, 8YSZ and BFO using DC-FS and MPFS.

Fig. 3(b) by an empty triangle). Fig. S6 shows the power density profiles for DC-FS and MPFS of 8 YSZ at $30 \mathrm{~V}$. It can be clearly seen that the generated power density in DC-FS is below the threshold value to activate the flash, in contrast to MPFS where the applied power is much higher and within the range at which the flash is activated. Indeed, 8YSZ ceramic can be flashed at voltages as low as $20 \mathrm{~V}$ using the MPFS technique, which demonstrates the efficiency of this novel method.

All in all, the experimental results reported in the present work suggest the potential applicability and versatility of the proposed novel MPFS technique. Ceramic materials characterized by different electrical conduction mechanisms are sintered at significantly lower temperatures than those necessary for conventional DCsystems at a given applied voltage. This result opens the venue to sinter materials in a more efficient way. Not only the flash onset temperature can be reduced but also hard or impossible to flash materials could be flashed by this newly developed MPFS method. Additionally, MPFS may avoid undesired local phenomena commonly occurring in conventional FS or reactive FS [25,29]. Essen- tially, sintering uniformity across the material is enhanced by a rotating electric field as opposed to a unidirectional field in conventional FS, which may magnify local phenomena in typically heterogeneous samples. Moreover, preferential current paths are less likely since, as observed for 8YSZ in the present work, the flash event occurs at notably lower voltages and, therefore, lower applied electric fields.

The possibility of achieving homogeneous sintering of complexshaped specimens by MPFS has been also explored in our work. Thus, 8YSZ 3D samples of different geometries, whose dimensions can be found in Fig. S1, were shaped and flash sintered using the Delta configuration. Fig. 4 shows details about a 8YSZ 3D triangle and clover-like specimens. The computational simulations in Fig. 4(a) and (e) suggest a homogeneous electric field distribution covering the whole surface of both samples. Additionally, the different shapes seem to contribute to different electric field distributions. The clover-like specimen accumulates charge density at the lobe border, which unveils that an adequate contour could modify the electric field distribution within the material for sintering 

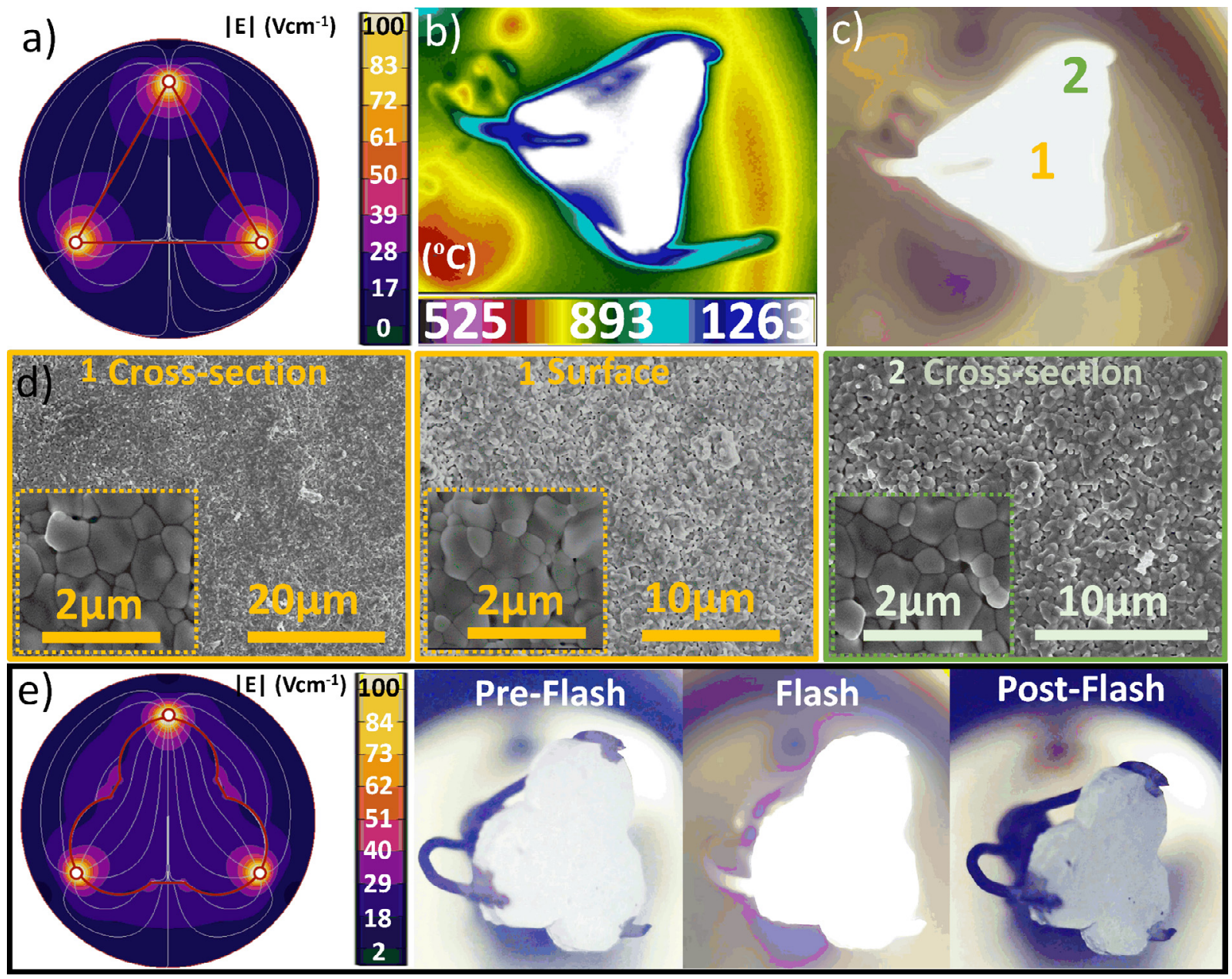

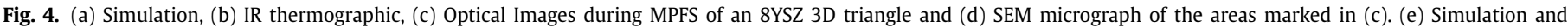
optical images of an 8YSZ 3D clover-like specimen at different stages during the MPFS experiment.

uniformity purposes. Nevertheless, in terms of densification uniformity, no significant differences were observed experimentally for the triangle and clover-like specimen as commented as follows. Fig. 4(b) and (c) includes the experimental results for the 3D triangle. Both IR thermographic and optical images suggest a homogeneous distribution of the flash characterized by uniform temperature profiles. Video S3 shows the MPFS process of the triangular specimen as recorded by the IR thermographic camera, which was carried out at $30 \mathrm{~V}$ and $2.5 \mathrm{~A}$. The flash takes place at a furnace temperature of $860{ }^{\circ} \mathrm{C}$. The small temperature gradients observed in Video S3 and Fig. 4(b) may be attributed to the cooling fluxes at the surface and edges of the sample, that are normally responsible of temperature gradients leading to heterogeneities, as well as the tilted position of the sample with respect to the IR camera. The typical strategies applied in conventional FS to alleviate these thermal gradient issues, such as running experiments in isothermal conditions with current-controlled ramps profiles combined with ultrafast heating rates and thermal insulators in the FS cavities could be definitely applied to MFPS [33]. Nevertheless, in this case, these reduced gradients are not even noticeable in the optical image and the SEM micrographs carried out near the electrodes and at the center of the sample, suggesting uniform densification with low porosity and an average grain size of approximately $800 \mathrm{~nm}$ (Fig. 4(d)). The density of the sample as measured by the Archimedes' method is $97 \%$, assuming $5.9 \mathrm{~g} \mathrm{~cm}^{-3}$ (Ref. Code 00-048-0224) as theoretical density. Similar results were obtained for the clover-like specimen plotted in Fig. 4(e) where the high de- gree of contraction and, therefore, densification (94\% measured by Archimedes') of the material is clearly evidenced in the optical images taken before (pre-flash) and after (post-flash) MPFS. For further details, see Video S4 that shows the MPFS event for the green compact at $30 \mathrm{~V}$ and $1.5 \mathrm{~A}$.

Very recently, Yang et al. reported the sintering of a complex gear-shaped 3YSZ specimen by conventional DC-FS [46]. DC voltage was applied between the two faces of the green body as this is the only possible option with conventional 2-electrodes FS. In contrast to the samples presented here, the specimen suffered from significant grain size and hardness gradients as well as porosity. The authors suggested that a greater homogeneity could be obtained by means of AC-FS. Even so, in single-phase AC systems the supplied power falls to zero twice in a cycle and therefore the power delivered, which may also contribute to heterogeneities. This limitation is partially avoided by the MPFS method since each phase delivers independent $120^{\circ}$ shifted AC voltage signals and the supplied power never drops to zero. Thus, this relatively constant power supply as well as the possibility of multiple electrode configurations to achieve different distributions of the electric field promote homogeneous sintering even in samples with complex geometries. Additionally, the combined use of MPFS and 3D printing can constitute the basis of a promising technique to produce ceramic specimens of complex shape.

Finally, we would like to emphasize the potential and versatility of MPFS. Fig. 5 shows the simulation results for a MPFS experiment using 6 phases. In comparison to the 3-phase simulation at the same voltage included in Fig. S3(a), not only the ap- 


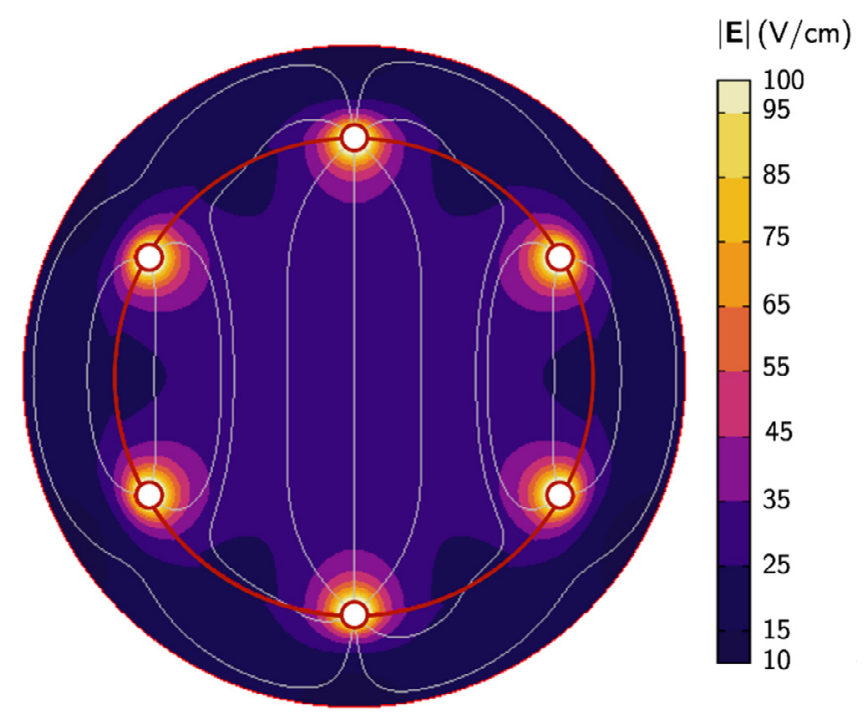

Fig. 5. Electric field distribution obtained from simulations of a MPFS system using 6 phases in Delta configuration. plied electric field is more homogeneous but also the covered surface due to the higher supplied power. As mentioned above, this has several practical implications for the sintering of materials in comparison to traditional FS, beside the advantageous possibility of a more convoluted configuration of the electrodes for the sintering of complex-shaped samples. Firstly, the applied voltage required for flash triggering is obviously lower in MPFS, which serves to mitigate localization phenomena and preferential current pathways commonly encountered in conventional FS. Secondly, at the same applied voltage, the supplied power is approximately proportional to the number of phases. In this way, samples with bigger volumes and more complicated geometries can be sintered at reduced temperatures, which boosts energy efficiency. Thus, MPFS has the potential to expand the domain of applications of ceramic materials.

\section{Conclusions}

This work constitutes the first proof-of-concept of Multi-Phase Flash Sintering (MPFS). It is demonstrated that 3-phase power supply can efficiently flash-sinter materials characterized by different

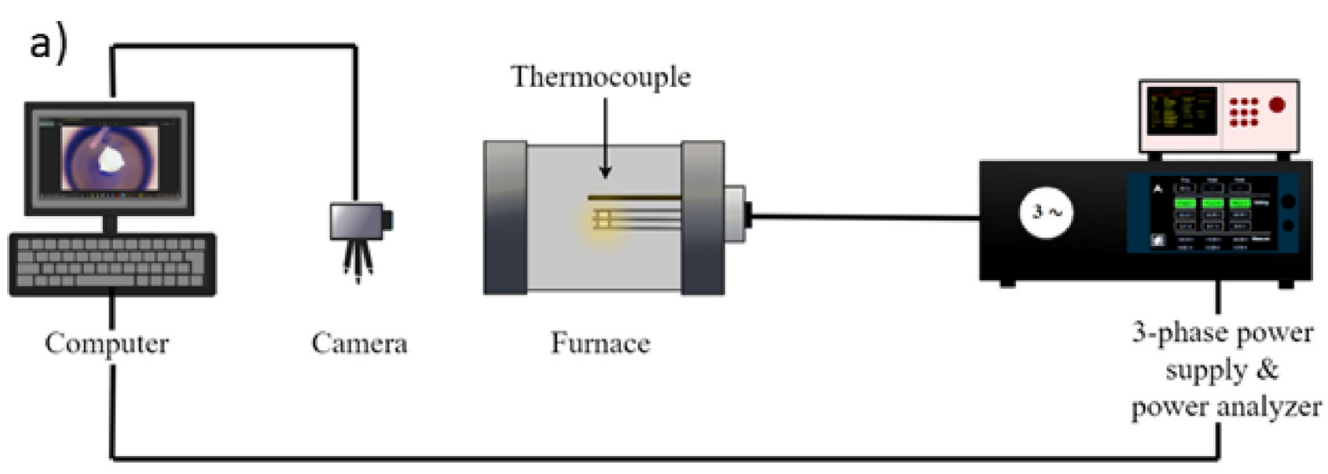

b)
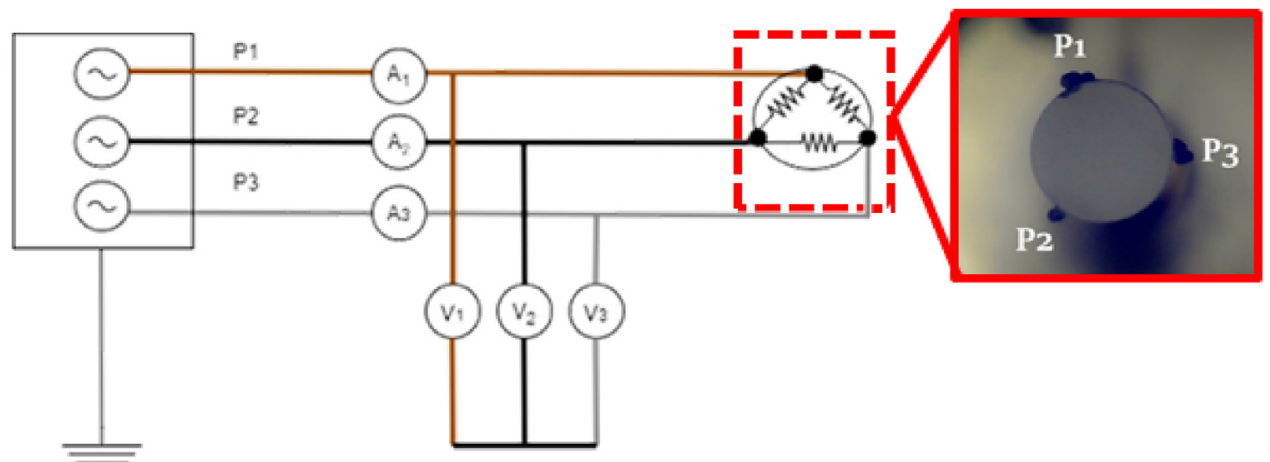

c)

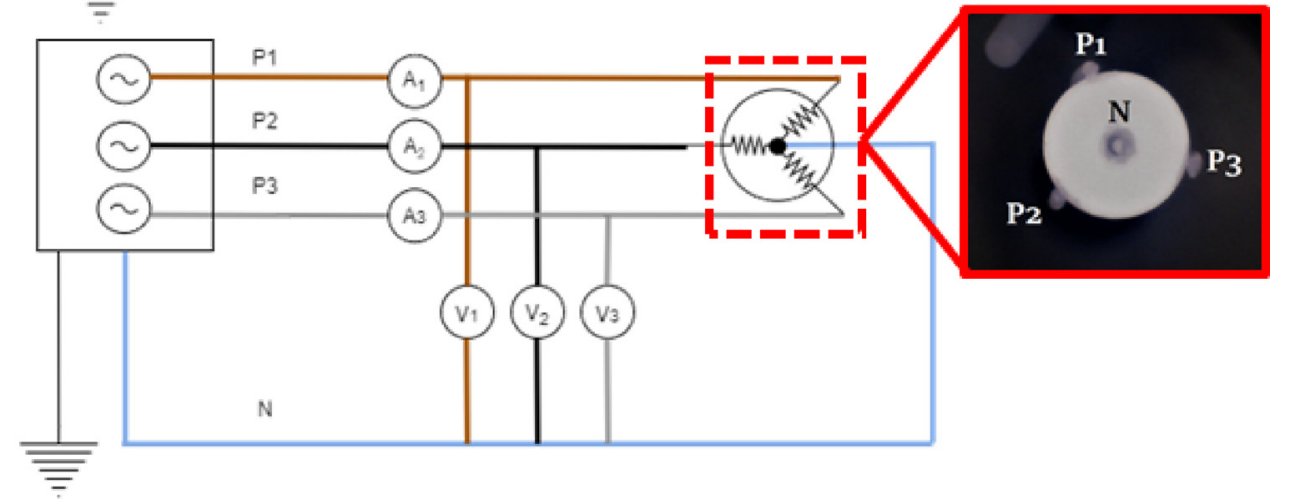

Scheme 1. (a) MPFS experimental setup and details of sample connection to a 3-phase power supply: (b) Delta and (c) Star configurations. 
electrical conduction mechanisms as well as 3D complex-shaped specimens.

Delta configurations exhibited a much more resilient behavior under electrical stress than Star setups. Delta settings reached a homogeneous flash in different complex samples. At the same time, in similar conditions, Star configurations showed local effects as preferential paths, limiting the homogeneity of the flash, and consequently the uniformity of the densification process. Therefore, Delta setups are the recommended settings in applications such as MPFS to mitigate the inception of hot spots and preferential pathways. The applicability of the MPFS technique to a wide range of materials and shapes is also demonstrated. Materials with different conductivities, i.e. $\mathrm{ZnO}, 8 \mathrm{YSZ}$ and $\mathrm{BFO}$, can be sintered in a matter of seconds at furnace temperatures lower than those used in conventional DC-FS under the same applied voltage. This is mainly due to the higher power densities applied in MPFS. Additionally, MPFS offers multiple possibilities of electrode configurations for the sintering of complex-shaped specimens. The most important practical implications for the enhanced sintering of materials by MPFS are: (1) the flash can be initiated at reduced voltages and (2) uniform electric fields over bigger volumes of complex geometries can be achieved. Hence, energy efficiency is improved and the typical issues related to the thermal management in conventional FS such as undesired local phenomena and preferential current pathways can be better coped in MPFS. Beside, the common strategies used in traditional FS to alleviate these thermal issues can be also applied to MPFS. Thus, specimens with complex geometries can be uniformly sintered, as demonstrated in the present work for the 3D triangle and clover-like specimens. These results pave the way to efficiently produce complex-shaped ceramic materials.

The newly developed MPFS method entails the next natural step in the future development of FS. The synergistic effect of increasing the number of phases in FS while reducing the onset flash temperature enables the efficient and homogeneous sintering of 3D complex-shaped ceramics, while keeping all other advantages of conventional FS. MPFS can be considered as an interesting methodology for industrial applications due to its smaller energy footmark in comparison to traditional sintering methodologies and even to conventional FS. Moreover, the combined use of 3D printing and MPFS can promote new applications of ceramic materials.

\section{Declaration of Competing Interest}

The authors declare that they have no known competing financial interests or personal relationships that could have appeared to influence the work reported in this paper.

\section{CRediT authorship contribution statement}

Sandra Molina-Molina: Investigation, Methodology, Writing review \& editing, Data curation. Eva Gil-González: Writing original draft, Visualization, Investigation. Francisco José DuránOlivencia: Methodology, Formal analysis. José Manuel Valverde: Conceptualization, Writing - review \& editing. Antonio Perejón: Writing - review \& editing, Supervision. Pedro E. SánchezJiménez: Conceptualization, Writing - review \& editing, Supervision. Luis A. Pérez-Maqueda: Conceptualization, Writing - review \& editing, Project administration.

\section{Acknowledgements}

The authors fully thank Ahmed Taibi for his valuable help on performing the experiments and discussion. This work has been supported by the Spanish Government Agency Ministerio de Ciencia, Innovación y Universidades and FEDER (projects CTQ201783602-C2-1-R and CTQ2017-83602-C2-2-R) and by Junta de An-
daluciá-Consejerıá de Conocimiento, Investigación y Universidad and FEDER (projects P18-FR-1087 and US-1262507). Financial support from project 201960E092 (INTRAMURAL-CSIC) is also acknowledged.

\section{Supplementary materials}

Supplementary material associated with this article can be found, in the online version, at doi:10.1016/j.apmt.2021.101274.

\section{References}

[1] O. Guillon, J. Gonzalez-Julian, B. Dargatz, T. Kessel, G. Schierning, J. Räthel, M. Herrmann, Field-assisted sintering technology/spark plasma sintering: mechanisms, materials, and technology developments, Adv. Eng. Mater. 16 (2014) 830-849, doi:10.1002/adem.201300409.

[2] M. Cologna, B. Rashkova, R. Raj, Flash sintering of nanograin zirconia in $<5$ s at $850^{\circ}$ C, J. Am. Ceram. Soc. 93 (2010) 3556-3559, doi:10.1111/j.1551-2916.2010. 04089.x.

[3] K. Naik, S.K. Jha, R. Raj, Correlations between conductivity, electroluminescence and flash sintering, Scr. Mater. 118 (2016) 1-4, doi:10.1016/j.scriptamat.2016.03. 001.

[4] R. Raj, Analysis of the power density at the onset of flash sintering, J. Am. Ceram. Soc. 99 (2016) 3226-3232, doi:10.1111/jace.14178.

[5] M. Yu, S. Grasso, R. Mckinnon, T. Saunders, M.J. Reece, Review of flash sintering: materials, mechanisms and modelling, Adv. Appl. Ceram. 116 (2017) 2460, doi:10.1080/17436753.2016.1251051.

[6] C.E.J. Dancer, Flash sintering of ceramic materials, Mater. Res. Express 3 (2016) 1-25, doi:10.1088/2053-1591/3/10/102001.

[7] R. Raj, Joule heating during flash-sintering, J. Eur. Ceram. Soc. 32 (2012) 22932301, doi:10.1016/j.jeurceramsoc.2012.02.030.

[8] J. Narayan, A new mechanism for field-assisted processing and flash sintering of materials, Scr. Mater. 69 (2013) 107-111, doi:10.1016/j.scriptamat.2013. 02.020 .

[9] R. Chaim, Liquid film capillary mechanism for densification of ceramic powders during flash sintering, Materials 9 (2016) 280 (Basel, Switzerland), doi:10. 3390/ma9040280.

[10] R. Raj, A. Kulkarni, J.M. Lebrun, S. Jha, Flash sintering: a new frontier in defect physics and materials science, MRS Bull. 46 (2021) 36-43, doi:10.1557| s43577-020-00011-1.

[11] M. Cologna, J.S.C. Francis, R. Raj, Field assisted and flash sintering of alumina and its relationship to conductivity and MgO-doping, J. Eur. Ceram. Soc. 31 (2011) 2827-2837, doi:10.1016/j.jeurceramsoc.2011.07.004.

[12] M. Biesuz, V.M. Sglavo, Flash sintering of ceramics, J. Eur. Ceram. Soc. 39 (2019) 115-143, doi:10.1016/j.jeurceramsoc.2018.08.048

[13] M.A.B. Wassel, L.A. Pérez-Maqueda, E. Gil-Gonzalez, H. Charalambous, A. Perejon, S.K. Jha, J. Okasinski, T. Tsakalakos, Anisotropic lattice expansion determined during flash sintering of $\mathrm{BiFeO}_{3}$ by in-situ energy-dispersive X-ray diffraction, Scr. Mater. 162 (2019) 268-291, doi:10.1016/j.scriptamat.2018.11. 028.

[14] S.K. Jha, H. Charalambous, H. Wang, X.L. Phuah, C. Mead, J. Okasinski, H. Wang, T. Tsakalakos, In-situ observation of oxygen mobility and abnormal lattice expansion in ceria during flash sintering, Ceram. Int. 44 (2018) 15362-15369, doi:10.1016/j.ceramint.2018.05.186.

[15] L.A. Perez-Maqueda, E. Gil-Gonzalez, A. Perejon, J.M. Lebrun, P.E. SanchezJimenez, R. Raj, Flash sintering of highly insulating nanostructured phase-pure $\mathrm{BiFeO}_{3}$, J. Am. Ceram. Soc. 100 (2017) 3365-3369, doi:10.1111/jace.14990.

[16] L.A. Perez-Maqueda, E. Gil-Gonzalez, M.A. Wassel, S.K. Jha, A. Perejon, H. Charalambous, J. Okasinski, P.E. Sanchez-Jimenez, T. Tsakalakos, Insight into the $\mathrm{BiFeO}_{3}$ flash sintering process by in-situ energy dispersive X-ray diffraction (ED-XRD), Ceram. Int. 45 (2019) 2828-2834, doi:10.1016/j.ceramint.2018.07. 293.

[17] E. Gil-González, A. Perejón, P.E. Sánchez-Jiménez, R. Raj, L.A. Pérez-Maqueda, Processing and properties of $\mathrm{Bi}_{0.98} \mathrm{R}_{0.02} \mathrm{FeO}_{3}(\mathrm{R}=\mathrm{La}, \mathrm{Sm}, \mathrm{Y})$ ceramics flash sintered at $\sim 650^{\circ} \mathrm{C}$ in $<5 \mathrm{~s}$, J. Am. Ceram. Soc. 103 (2020) 136-144, doi:10.1111/ jace.16718.

[18] N. Shomrat, S. Baltianski, C.A. Randall, Y. Tsur, Flash sintering of potassiumniobate, J. Eur. Ceram. Soc. 35 (2015) 2209-2213, doi:10.1016/j.jeurceramsoc. 2015.01.017.

[19] T. Clemenceau, N. Andriamady, P. Kumar, M.K.A. Badran, V. Avila, K. Dahl, M. Hopkins, X. Vendrell, D. Marshall, R. Raj, Flash sintering of Li-ion conducting ceramic in a few seconds at $850^{\circ} \mathrm{C}$, Scr. Mater. 172 (2019) 1-5, doi:10.1016/ j.scriptamat.2019.06.038.

[20] J. Li, J. Cho, J. Ding, H. Charalambous, S. Xue, H. Wang, X.L. Phuah, J. Jian, X. Wang, C. Ophus, T. Tsakalakos, R.E. García, A.K. Mukherjee, N. Bernstein, C.S. Hellberg, H. Wang, X. Zhang, Nanoscale stacking fault-assisted room temperature plasticity in flash-sintered $\mathrm{TiO}_{2}$, Sci. Adv. 5 (2019) eaaw5519, doi:10. 1126/sciadv.aaw5519.

[21] K. Klauke, B. Kayaalp, M. Biesuz, A. Iannaci, V.M. Sglavo, M. D’Arienzo, S. Lee, J. Seo, W. Jung, S. Mascotto, Enhancement of the $\mathrm{SrTiO}_{3}$ surface reactivity by exposure to electric fields, ChemNanoMat 5 (2019) 948-956, doi:10.1002 cnma.201900201. 
[22] H. Zhou, X. Li, Y. Zhu, J. Liu, A. Wu, G. Ma, X. Wang, Z. Jia, L. Wang, Review of flash sintering with strong electric field, High Volt. (2021), doi:10.1049/hve2. 12080.

[23] J. Nie, Y. Zhang, J.M. Chan, R. Huang, J. Luo, Water-assisted flash sintering: flashing $\mathrm{ZnO}$ at room temperature to achieve 298 density in seconds, Scr. Mater. 142 (2018) 79-82, doi:10.1016/j.scriptamat.2017.08.032.

[24] Y. Zhang, J. Luo, Promoting the flash sintering of $\mathrm{ZnO}$ in reduced atmospheres to achieve nearly full densities at furnace temperatures of $<120{ }^{\circ} \mathrm{C}$, Scr. Mater. 106 (2015) 26-29, doi:10.1016/j.scriptamat.2015.04.027.

[25] E. Gil-González, A. Perejón, P.E. Sánchez-Jiménez, MJ. Sayagués, R. Raj, L.A. Pérez-Maqueda, Phase-pure $\mathrm{BiFeO}_{3}$ produced by reaction flash-sintering of $\mathrm{Bi}_{2} \mathrm{O}_{3}$ and $\mathrm{Fe}_{2} \mathrm{O}_{3}$, J. Mater. Chem. A 6 (2018) 5356-5366, doi:10.1039/ c7ta09239c.

[26] E. Gil-González, A. Perejón, P.E. Sánchez-Jiménez, D. Román-González, L.A. Pérez-Maqueda, Control of experimental conditions in reaction flashsintering of complex stoichiometry ceramics, Ceram. Int. 46 (2020) 2941329420, doi:10.1016/j.ceramint.2020.05.091.

[27] H.R. Mao, R.F. Guo, Y. Cao, S.B. Jin, X.M. Qiu, P. Shen, Ultrafast densification of high-entropy oxide $\left(\mathrm{La}_{0.2} \mathrm{Nd}_{0.2} \mathrm{Sm}_{0.2} \mathrm{Eu}_{0.2} \mathrm{Gd}_{0.2}\right)_{2} \mathrm{Zr}_{2} \mathrm{O}_{7}$ by reactive flash sintering, J. Eur. Ceram. Soc. 41 (2021) 2855-2860, doi:10.1016/j.jeurceramsoc.2020. 11.052.

[28] V. Avila, R. Raj, Reactive flash sintering of powders of four constituents into a single phase of a complex oxide in a few seconds below $700^{\circ} \mathrm{C}, \mathrm{J}$. Am. Ceram. Soc. 102 (2019) 6443-6448, doi:10.1111/jace.16625

[29] A. Taibi, S. Chaguetmi, P.E. Sánchez-Jiménez, A. Perejón, J.E. García, H. Satha, L.A. Pérez-Maqueda, Pure perovskite $\mathrm{BiFeO}_{3}-\mathrm{BaTiO}_{3}$ ceramics prepared by reaction flash sintering of $\mathrm{Bi}_{2} \mathrm{O}_{3}-\mathrm{Fe}_{2} \mathrm{O}_{3}-\mathrm{BaTiO}_{3}$ mixed powders, Ceram. Int. (2021), doi:10.1016/j.ceramint.2021.06.108.

[30] T.P. Mishra, C. Lenser, R. Raj, O. Guillon, M. Bram, Development of a processing map for safe flash sintering of gadolinium-doped ceria, J. Am. Ceram. Soc. (2021) 1-13, doi:10.1111/jace.17847.

[31] H. Charalambous, S.K. Jha, H. Wang, X.L. Phuah, H. Wang, T. Tsakalakos, Inhomogeneous reduction and its relation to grain growth of titania during flash sintering, Scr. Mater. 155 (2018) 37-40, doi:10.1016/j.scriptamat.2018.06.017.

[32] M. Biesuz, V.M. Sglavo, Current-induced abnormal and oriented grain growth in corundum upon flash sintering, Scr. Mater. 150 (2018) 82-86, doi:10.1016/j. scriptamat.2018.03.004.

[33] G.M. Jones, M. Biesuz, W. Ji, S.F. John, C. Grimley, C. Manière, C.EJ. Dancer, Promoting microstructural homogeneity during flash sintering of ceramics through thermal management, MRS Bull. 46 (2021) 59-66, doi:10.1557/ s43577-020-00010-2.

[34] H. Charalambous, S.K. Jha, K.H. Christian, R.T. Lay, T. Tsakalakos, Flash sintering using controlled current ramp, J. Eur. Ceram. Soc. 38 (2018) 3689-3693, doi:10. 1016/j.jeurceramsoc.2018.04.003.

[35] X.L. Phuah, H. Wang H. Charalambous, S.K. Jha, T. Tsakalakos, X Zhang $\mathrm{H}$. Wang, Comparison of the grain growth behavior and defect structures of flash sintered $\mathrm{ZnO}$ with and without controlled current ramp, Scr. Mater. 162 (2019) 251-255, doi:10.1016/j.scriptamat.2018.11.009.

[36] I.R. Lavagnini, J.V. Campos, J.A. Ferreira, E.M.J.A. Pallone, Microstructural evolution of 3YSZ flash-sintered with current ramp control, J. Am. Ceram. Soc. 103 (2020) 3493-3499, doi:10.1111/jace.17037.
[37] C. Manière, F. Borie, S. Marinel, Impact of convection and radiation on direct/hybrid heating stability of field assisted sintering, J. Manuf. Process. 56 (2020) 147-157, doi:10.1016/j.jmapro.2020.04.075.

[38] M. Biesuz, L. Pinter, T. Saunders, M. Reece, J. Binner, V.M. Sglavo, S. Grasso, Investigation of electrochemical, optical and thermal effects during flash sintering of 8YSZ, Materials 11 (2018). 10.3390/ma11071214.

[39] W. Qin, H. Majidi, J. Yun, K. van Benthem, Electrode effects on microstructure formation during FLASH sintering of yttrium-stabilized zirconia, J. Am. Ceram. Soc. 99 (2016) 2253-2259, doi:10.1111/jace.14234.

[40] C.A. Grimley, A.L.G. Prette, E.C. Dickey, Effect of boundary conditions on reduction during early stage flash sintering of YSZ, Acta Mater. 174 (2019) 271-278, doi:10.1016/j.actamat.2019.05.001.

[41] L. Guan, J. Li, X. Song, J. Bao, T. Jiang, Graphite assisted flash sintering of $\mathrm{Sm}_{2} \mathrm{O}_{3}$ doped $\mathrm{CeO}_{2}$ ceramics at the onset temperature of $25^{\circ} \mathrm{C}$, Scr. Mater. 159 (2019) 72-75, doi:10.1016/j.scriptamat.2018.09.014.

[42] G. Fele, M. Biesuz, P. Bettotti, R. Moreno, V.M. Sglavo, Flash sintering of yttriastabilized zirconia/graphene nano-platelets composite, Ceram. Int. 46 (2020) 23266-23270, doi:10.1016/j.ceramint.2020.06.008

[43] X.L. Phuah, H. Wang, Z. Qi, S. Misra, M. Kalaswad, H. Wang, Field-assisted heating of Gd-doped ceria thin film, J. Am. Ceram. Soc. 103 (2020) 2309-2314, doi:10.1111/jace.16949.

[44] V. Avila, R. Raj, Flash sintering of ceramic films: the influence of surface to volume ratio, J. Am. Ceram. Soc. 102 (2019) 3063-3069, doi:10.1111/jace.16298.

[45] Z. Chen, Z. Li, J. Li, C.C. Liu, C. Lao, Y. Fu, C.C. Liu, Y. Li, P. Wang, Y. He, 3D printing of ceramics: a review, J. Eur. Ceram. Soc. 39 (2019) 661-687, doi:10. 1016/j.jeurceramsoc.2018.11.013.

[46] B. Yang, J. Cho, X.L. Phuah, H. Wang, X. Zhang, Flash sintering of additively manufactured 3YSZ gears, J. Am. Ceram. Soc. 104 (2021) 3828-3832, doi:10. $1111 /$ jace. 17835.

[47] A. Perejón, N. Murafa, P.E. Sánchez-Jiménez, J.M. Criado, J. Subrt, M.J. Diánez, L.A. Pérez-Maqueda, Direct mechanosynthesis of pure $\mathrm{BiFeO}_{3}$ perovskite nanoparticles: reaction mechanism, J. Mater. Chem. C 1 (2013) 3551-3562, doi:10.1039/C3TC30446A

[48] S.K. Jha, K. Terauds, J.M. Lebrun, R. Raj, Beyond flash sintering in $3 \mathrm{~mol} \%$ yttria stabilized zirconia, J. Ceram. Soc. Jpn. 124 (2016) 283-288, doi:10.2109/jcersj2. 15248.

[49] R.I. Todd, E. Zapata-Solvas, R.S. Bonilla, T. Sneddon, P.R. Wilshaw, Electrical characteristics of flash sintering: thermal runaway of Joule heating, J. Eur. Ceram. Soc. 35 (2015) 1865-1877, doi:10.1016/j.jeurceramsoc.2014.12.022.

[50] Y. Zhang, J.I. Jung, J. Luo, Thermal runaway, flash sintering and asymmetrical microstructural development of $\mathrm{ZnO}$ and $\mathrm{ZnO}-\mathrm{Bi}_{2} \mathrm{O}_{3}$ under direct currents, Acta Mater. 94 (2015) 87-100, doi:10.1016/j.actamat.2015.04.018.

[51] M.C. Steil, D. Marinha, Y. Aman, J.R.C. Gomes, M. Kleitz, From conventional ac flash-sintering of YSZ to hyper-flash and double flash, J. Eur. Ceram. Soc. 33 (2013) 2093-2101, doi:10.1016/j.jeurceramsoc.2013.03.019.

[52] Y. Dong, I.W. Chen, Predicting the onset of flash sintering, J. Am. Ceram. Soc. 98 (2015) 2333-2335, doi:10.1111/jace.13679. 